\title{
La Glossodiversité, prolongement de la biodiversité chez Homo Sapiens
}

\section{Gilbert Dalgalian}

\section{(2) OpenEdition}

\section{Journals}

Édition électronique

URL : http://journals.openedition.org/esp/2786

DOI : $10.4000 /$ esp. 2786

ISSN : 2532-0319

Éditeur

Centre d'Information sur l'Éducation Bilingue et Plurilingue

\section{Édition imprimée}

Date de publication : 1 décembre 2013

Pagination : 81-85

ISSN : 1127-266X

\section{Référence électronique}

Gilbert Dalgalian, «La Glossodiversité, prolongement de la biodiversité chez Homo Sapiens »,

Éducation et sociétés plurilingues [En ligne], 35 | 2013, mis en ligne le 01 février 2020, consulté le 17

février 2021. URL : http://journals.openedition.org/esp/2786; DOI : https://doi.org/10.4000/esp.2786 


\section{LA GLOSSODIVERSITÉ, PROLONGEMENT DE LA BIODIVERSITÉ CHEZ HOMO SAPIENS (1)}

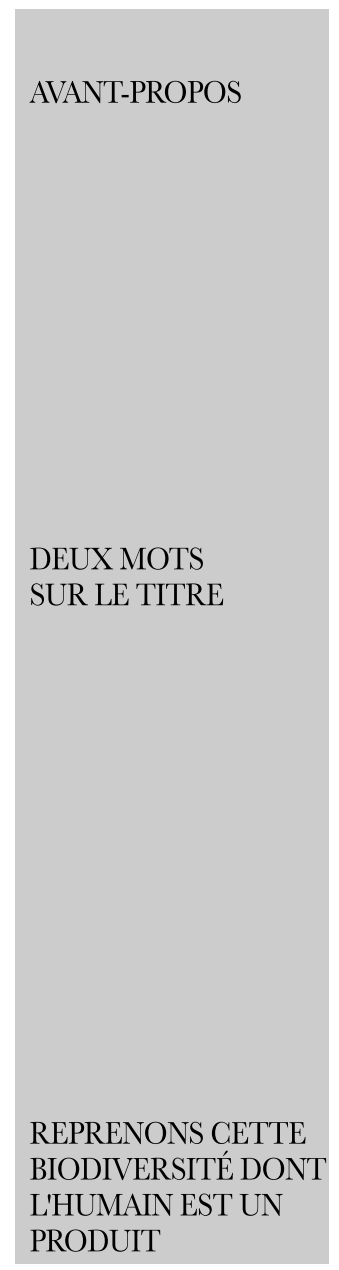

\section{Gilbert Dalgalian}

A vant d'expliciter ce titre, deux mots sur mon parcours, étroitement lié au thème. Je suis psycholinguiste, plus connu pour ma spécialisation sur les apprentissages précoces et le bilinguisme précoce. Ce qui explique mon engagement pour l'enseignement des langues régionales.

Mais je suis passé peu à peu de la construction du langage chez l'enfant à l'émergence du langage dans l'espèce, autrement dit à l'invention fondatrice d'Homo Sapiens. Ce qui m'a obligatoirement conduit vers l'anthropologie, la paléoantropologie et la génétique. Cette pluridisciplinarité correspond aux multiples dimensions de l'humain, à la multiplicité de facteurs qui ont fait advenir Homo Sapiens. Et que je vais rappeler brièvement.

Je vais me focaliser ici sur la filiation entre la biodiversité dont Sapiens est le produit et la diversité des langues et des cultures dont Sapiens est l'inventeur et le véhicule.

Plusieurs auteurs ont pressenti cette filiation et l'ont curieusement nommée 'biodiversité linguistique', comme si les faits observés étaient de même nature. Or, s'il y a filiation, il y a aussi une différence qualitative, suffisante en tous cas pour inventer un néologisme. Ce que j'ai fait en proposant le terme de 'Glossodiversité' dans mon premier livre (Reconstruire l'éducation, réédité en 2012), terme repris et approfondi dans mon dernier livre, Capitalisme à l'agonie: quel avenir pour Homo Sapiens? L'Harmattan, 2012 (voir le

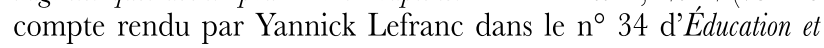
Sociétés Plurilingues).

Dans Glossodiversité j'englobe évidemment la diversité culturelle dont les langues sont porteuses.

Ici je serai sommaire et forcément succinct. Car je ne prétends pas me substituer à Yves Coppens, notre grand paléoanthropologue, mais je vais m'en inspirer. Comme je vais m'appuyer sur Albert Jacquard pour les dimensions génétiques. 
La Glossodiversité, prolongement de la biodiversité chez Homo Sapiens

G. DALGALIAN

L'HYPOTHÈSE FORTE, LA VOICI
Leurs disciplines se recoupent et conduisent à une même hypothèse forte. Pourquoi hypothèse? Et pourquoi forte? Hypothèse, parce qu'il manque encore quelques fossiles humains, quelques habitats rudimentaires, quelques outils préhistoriques qui viendront confirmer et peut-être nuancer cette hypothèse.

Mais c'est une hypothèse forte. D'autant plus forte que, sans elle, on ne comprend ni le singulier paradoxe du bébé humain (j'y viens), ni la prolifération époustouflante qui a saisi Sapiens et probablement déjà Erectus depuis les origines.

En effet, comment comprendre qu'une telle profusion de profils ethniques, linguistiques, culturels, des techniques de vie et de survie, des croyances et des rituels - et de profils individuels ait proliféré à ce degré inouï dans l'espèce humaine? Il y a nécessairement un facteur déclencheur, un moment privilégié, un saut qualitatif. Est-ce la continuation sans heurts ni ruptures de la biodiversité?

La première probablement - et la plus formidable certainement des auto-inventions qui ont précédé l'émergence d'Homo Sapiens fut le passage à la bipédie, suite à la perte de l'habitat forestier et de la vie dans les arbres. Ce fut la naissance d'Homo Erectus.

La confrontation brutale et durable avec la savane, les déserts, les vastes étendues, ont changé l'homme et la femme: redressement de la colonne vertébrale, verticalisation du bassin, descente du larynx, repositionnement du crâne, gain de volume du cerveau et nourriture diversifice, affinement des sens pour observer et aller plus loin, conquête de nouveaux outils, d'habiletés corporelles inédites, longtemps avant le feu.

Mais la conséquence la plus cruciale de cette très longue métamorphose va concerner la femme Erectus: avec son bassin désormais plus fin et verticalisé, elle ne peut plus garder le bébé jusqu'au terme d'une grossesse qui était à l'origine plus longue de plusieurs semaines ou mois (peut-être ne saura-t-on jamais exactement de combien plus longue). La grossesse va finir par se caler sur les neuf mois que nous connaissons.

Voici ce que dit Yves Coppens dans L'Histoire de l'Homme (pp. 5657):

«... ce raccourcissement de la distance entre l'articulation sacroiliaque et l'articulation de la hanche qui a l'avantage de décroître le moment de rotation créé par le poids du corps sur la hanche, a [aussi] le désavantage de réduire la taille de la cavité pelvienne, entrainant une parturition ventrale ... contrairement à celle des grands singes qui est dorsale ... 
La Glossodiversité, prolongement de la biodiversité chez Homo Sapiens

G. DALGALIAN
«Cette constatation de l'apparition très précoce du mode humain de parturition souligne ses liens avec la bipédie et non pas, comme on l'a souvent écrit, avec l'agrandissement du cerveau». On voit que la paléontologie a nettement identifié à la fois la nouvelle anatomie de l'accouchée, la nouvelle physiologie de l'accouchement et - le plus important pour l'émergence de Sapiens - la durée plus courte de la grossesse. Depuis quand? Et de combien de mois? Nous ne pourrons probablement pas atteindre avant longtemps la réponse à ces deux questions. Une chose est acquise: la femme Erectus, en se redressant, ne pouvait plus garder jusqu'à son terme un bébé dont la tête était désormais trop grosse pour un bassin verticalisé et plus étroit.

Avec cette conséquence jusqu'à ce jour et pour longtemps: depuis Erectus - au plus tard - nous sommes tous des prématurés. Et parce que prématuré, le cerveau du bébé humain, bien que parfaitement doté de milliards de neurones à la naissance, arrive au monde totalement immature. Il ne possède qu'un seul savoir-faire, contrairement aux autres espèces beaucoup mieux dotées pour la survie: il n'a que le réflexe de la tétée.

C'est cela le paradoxe du bébé humain: un cerveau richement doté, mais immature, qui va connaître la plus longue éducabilité de toutes les espèces vivantes. Et pour cette raison le petit Erectus, puis Sapiens, va subir comme jamais auparavant la pression ou l'influence de l'environnement: naturel, social, technique et culturel. Environnement partout différent et toujours changeant! Avec l'impact le plus long et le plus marquant entre zéro et sept ans, l'âge des acquisitions fondamentales.

C'est donc l'évidence d'une gestation plus courte et d'une naissance plus précoce qui a conduit Albert Jacquard à formuler distinctement cette hypothèse forte sans laquelle on ne peut comprendre l'infinie diversification des morphotypes ou variantes d'Homo au fil des âges. Ni par la suite celle des ethnies, des langues et des cultures et même des profils individuels.

Le regretté Albert Jacquart aurait pu, s'il était parmi nous, développer davantage les implications de cette prématurité. Je vais m'y efforcer le plus clairement possible..

Depuis cette mutation fondatrice, notre immaturité à la naissance nous a ouvert le plus vaste champ de potentialités d'acquisitions, d'apprentissages et d'inventions, que seule la combinaison unique d'un cerveau inachevé mais bien doté avec une très longue épigenèse aura fait surgir dans le règne du vivant. L'extrême vulnérabilité de nouveau-né humain est compensée par une très longue prise en charge par une mère ou un clan qui lui assurent la survie 
La Glossodiversité, prolongement de la biodiversité chez Homo Sapiens

G. DALGALIAN et la transmission des savoirs indispensables à son autonomie.

Notre éducabilité unique et exclusive dans le monde vivant est le bénéfice induit de notre naissance en tant que prématurés. Et qui induit, par la suite, notre longue sujétion à l'environnement. C'est la pression de l'environnement qui transforme nos potentialités génétiques en réalités de savoirs et de savoir-faire.

Le langage, mais aussi les nouveaux outils, les mythes, les musiques, les techniques et les religions ne sont pas le premier saut qualitatif; ils sont la conséquence et les retombées de cette éducabilité inédite d'un prématuré. Si Homo n'a jamais cessé de naître au fil de ses inventions, c'est bien à partir de cet accouchement précoce qu'il connaîtra sa véritable accélération en Sapiens Sapiens. Toujours sous pression de l'environnement.

Que nous restions si longtemps ouverts à des myriades d'acquistions précoces et par la suite à de multiples reconfigurations neuronales, c'est bien dû essentiellement au fait de cette très archaïque naissance prématurée il y a plus d'un million d'années. On doit s'étonner que l'on n'ait pas pressenti plus tôt l'origine de cette ouverture infinie de l'humain et de l'humain seul à ces milliards d'acquisitions qui ont fait Sapiens! Comme une étrange myopie sur nous-mêmes ... et il faudra bien s'interroger longuement sur cette myopie-là.

On peut dire que sa naissance avec ce cerveau immature a permis à Homo d'échapper à la dictature des gènes pour accéder non pas à une nouvelle dictature de l'environnement, mais à une nouvelle ère d'interactions intenses et permanentes entre les gènes et l'environnement.

C'est ce changement du rapport à l'environnement qui s'intensifie et va déterminer les nouvelles évolutions inédites de l'espèce Homo. Comment imaginer en effet qu'avec ses nouveaux outils et capacités, Sapiens n'ait pas prolongé l'évolution darwinienne sur des chemins inédits? Evolutions qui ne dépendent plus exclusivement du patrimoine génétique, mais viennent au contraire le compléter par un nouveau patrimoine d'innovations et d'apprentissages acquis et transmis de génération en génération.

Entre biodiversité et glossodiversité il y a en même temps filiation essentielle et rupture dans les formes et les évolutions.

Si le maintien de la biodiversité est à terme la condition de la survie physique de l'humanité, la glossodiversité et son corollaire, la diversité intellectuelle, sont les facteurs fondamentaux de notre créativité et de nos adaptations futures, de notre aptitude même à trouver des solutions collectives aux urgences d'aujourd'hui et de 
La Glossodiversité, prolongement de la biodiversité chez Homo Sapiens

G. DALGaLIAN demain.

Le dernier saut qualitatif qui reste à inventer vers une civilisation solidaire, si Sapiens veut survivre, n'est pas à espérer d'un nivellement culturel et d'une uniformisation linguistique qui sont autant de régressions et d'apauvrissements - et je ne pense pas seulement à l'anglais dominant - mais ce saut qualitatif est à rechercher dans nos enrichissements mutuels et notre respect de l'Autre: le respect de nos diversités est le test de notre aptitude à l'auto-invention collective. Cela passe par la promotion et la transmission de toutes les langues et cultures. N'oublions jamais que le mépris colonial et post-colonial se sont manifestés entre autres dans la morgue linguistique et culturelle des dominants. Cela a commencé au sein même des États, avant de s'exporter dans les empires coloniaux. L'ethnocentrisme imprègne encore largement les mentalités, malgré les nombreuses tentatives de désintoxication dont nous sommes redevables aux ethnologues, aux sociologues, aux philosophes et aux linguistes.

En conclusion et pour le dire sans détours, la révision de nos repères et la réforme de nos mentalités exigent la reconnaissance active de ce qui a fait de nous des humains: la diversité, le métissage et l'invention dès la préhistoire de la solidarité organisée, et aujourd'hui en net recul. Au point qu'elle est à repenser de fond en comble.

La glossodiversité et la diversité intellectuelle sont une condition sine qua non de la démocratie à inventer. Pour une civilisation qui tourne le dos à l'actuelle barbarie émergente.

\section{NOTE}

(1) Conférence donnée au CREDD'O à Eyragues, 28 septembre 2013. Colloque d'Automne: "Sur les sentiers de la culture provençale". 